Research Article

\title{
Research on Virtual Reality Interactive Teaching under the Environment of Big Data
}

\author{
Ziyan Yin $\mathbb{D}^{1}$ and Sang-Bing Tsai $\mathbb{D}^{2}$ \\ ${ }^{1}$ Hebei Petroleum University of Technology, Chengde 067000, China \\ ${ }^{2}$ Regional Green Economy Development Research Center, School of Business, Wuyi University, Nanping, China \\ Correspondence should be addressed to Ziyan Yin; yanziyin0305@126.com and Sang-Bing Tsai; sangbing@hotmail.com
}

Received 24 September 2021; Revised 22 October 2021; Accepted 1 November 2021; Published 10 December 2021

Academic Editor: Gengxin Sun

Copyright (c) 2021 Ziyan Yin and Sang-Bing Tsai. This is an open access article distributed under the Creative Commons Attribution License, which permits unrestricted use, distribution, and reproduction in any medium, provided the original work is properly cited.

\begin{abstract}
After decades of progress in virtual reality, the key technologies among them have reached the foundation to support the development of the virtual reality industry. Immersive virtual reality classroom is a bold attempt to combine present-day information science and technology with innovative teaching concepts, which inherits the characteristics of existing online education such as short and compact, large-scale, and free and open. Combined with immersive virtual reality technology, virtual reality classrooms will present strong. The virtual reality classroom will present a strong sense of immersion, interactivity, and conceptualization. This paper elaborates on the development history of virtual reality and its future development direction from the technical perspective and educational perspective, respectively. Taking Civic Science course as an example, after analyzing the feasibility and development significance of immersive virtual reality interactive teaching classroom in detail, the design scheme of immersive virtual reality classroom is proposed, the characteristics and advantages of virtual immersive virtual reality classroom are discussed, and the application of immersive virtual reality technology to classroom teaching is explored. Combined with the constructed virtual reality classroom, the testing, analysis, and evaluation work should be completed and corresponding improvements should be made to better meet the personalized learning needs of learners.
\end{abstract}

\section{Introduction}

With the rapid development of the Internet, the Internet has become a new position for the ideological and political education work of college students, and "Internet ideological and political education" has been born because of the Internet. In the field of education, virtual reality technology has become an important content and method for college students' ideological and political education. As far as the education field is concerned, virtual reality technology, as one of the important modern teaching tools, is constantly promoting the development and innovation of education methods. Contemporary education advocates the cultivation of students' independent learning ability and active exploration ability and requires educational institutions to teach with students as the main body. However, most schools today still use the traditional boring and mechanical teaching mode, leaving little room for students to explore. Students always lack initiative in the learning process, which leads to a decrease in teachers' teaching efficiency. Virtual reality-based educational products can greatly help to alleviate these problems: virtual space can provide a more vivid and creative teaching space, using multisensory channels to deepen the key impressions, enhance students' independent learning ability, cultivate students' interest in learning, and develop their creative thinking.

As a key technology of the new round of technological revolution, big data can be used as a perspective to study the ideological and political work of colleges and universities and can promote the paradigm transformation of such research work by deeply integrating it with big data to provide a paradigm for interdisciplinary analysis [1]; big data gradually evolves into a driving force for development in various fields, and using it to study the affairs of colleges and 
universities can accelerate the segmentation, stratification, and triage of the object and expand the theory of personalized education. Current virtual reality technology involves theories related to numerous disciplines and technologies, which include artificial intelligence, image processing, pattern recognition, graphics, and many other high-performance computing technologies. It expands the thinking and boundaries of personalized education theory and helps accelerate the reform of university education in the new era. By clearly defining relevant core concepts, it can promote the consolidation of the theoretical system of ideological and political work in colleges and universities and based on path innovation can change the concept of ideological and political work in colleges and universities; by integrating quantitative and qualitative methods, it can break through the solidified path of ideological and political work in colleges and universities and provide theoretical basis and the reference model for other academic researches by combining its relevant relationships. The virtual reality interactive teaching system provides an open learning environment [2], creates a pleasant and relaxed learning environment for students, promotes students' hands-on and operational abilities through various interactive forms, and cultivates students to become independent and efficient learners, thus further enhancing learning efficiency and learning experience. In the face of students' different cognitive abilities, the interactive learning form supported by virtual reality as technology can enable students to get timely and positive learning feedback, encourage them to try boldly and feel positive learning experience in the process of completing learning plans, and cultivate students' learning self-confidence, which is completely different from traditional teaching classrooms. Students are constantly cognizing things and trying to explore unknown knowledge in interactive learning, which in turn stimulates their imagination [3]. The use of virtual reality technology not only creates a virtual but realistic learning situation for students but also effectively helps teachers to enhance the diversity and expressiveness of teaching methods and improve the effectiveness and efficiency of their teaching, so the study of virtual reality interactive learning forms also has certain positive significance for the development of the education field.

\section{Related Work}

At present, foreign virtual reality technology has a wide range of applications, including military teaching, medical teaching, sports training, and other areas of teaching, virtual reality technology for educators to open up a broader vision, providing a more effective form of education. The United States is a more developed country in virtual reality technology, and its virtual reality technology level is higher than the world average technology level. U.S. research in this field focuses on four areas: student perception, student interface, backend software, and hardware.

In the literature [4], a boom-based virtual scene was investigated for the drawbacks of the low resolution of HMD systems, too heavy helmets, and eye discomfort, but it still did not solve the limitations of single-student virtual environments and eye discomfort due to too close screens. The literature [5] proposed the concept of a cavernous automated virtual environment with multistudent support and successfully solved the problem of eye discomfort for students at that time, which could provide users with unprecedented immersion. Since then, immersive virtual reality environments have incorporated projection elements, where students can interact with virtual objects in the projection plane. The literature [6] proposes a single projection table at a 45-degree angle, and the whole Immersa Desk system resembles a design table, facilitating students' autonomous interaction. In the same year, Virtual Reality Technology was published, which is also known as the first book on virtual reality. In addition, the literature [7] proposes the PowerWall system, which consists of multiple projection planes interlaced with each other, mainly for the visualization of scientific data. Since virtual systems with multiple projection planes can provide a larger field of view and higher immersion, virtual systems with five- and sixsided projection have emerged one after another, mainly used in virtual theaters for multiperson viewing. In terms of specific applications of the technology, the literature [8] presents a framework and experiments on immersive virtual reality-based learning operations techniques. The literature [9] details the structure and functionality of a cube immersion system with a length, width, and height of 3 meters. The literature [10] addresses the problem that gesture manipulation in student immersion systems is prone to fatigue and enhances students' experience satisfaction and perceived applicability based on the evaluation of simulation cycles and sensor update cycle times. The literature [11] demonstrated that users have a sense of presence in immersive virtual reality, creating the experience of mistaking scenarios and events as real occurrences. The literature [12] organizes research on the underlying theory and algorithms of virtual reality to facilitate the construction of virtual scenarios and the application of human-computer interaction design. The literature [13] investigated the sense of presence in virtual reality technology and focused on experimental testing of the sense of depth. The literature [14] successfully implements a multiprojection type of virtual simulation environment. In terms of technological development, the literature [15] studied a three-channel curved screen virtual reality system for students at a lower cost. The literature [16] specifically analyzed the factors related to the comfort experience in immersive virtual reality systems and proposed a position tracker data filtering algorithm. This algorithm effectively improves the real-time tracking performance and display of the position tracker successfully mitigates the user's dizziness and discomfort and facilitates the development of immersive virtual reality systems. The literature [17] identifies and analyzes the specific causes of positioning failure in immersive virtual reality systems and successfully conducts an innovative study on the fault tolerance of the system.

In terms of teaching applications, the literature [18] implemented a virtual chemistry laboratory using virtual reality technology, where students can observe chemical 
phenomena and perform virtual chemistry experiments. The literature [19] designed and developed a virtual teaching platform based on a circuit course, where students can complete the design of circuit diagrams and learn knowledge through virtual interactive sessions, while teachers can analyze students' learning directly on the platform. The virtual laboratory designed and developed in the literature [20] enables students to learn thermodynamics, astrophysics, and energetic environment in a virtual environment. The virtual laboratory developed in the literature [21] includes physics content such as optics and wave dynamics.

\section{Research on Virtual Reality Interactive Teaching of Civics Course in the Big Data Environment}

\subsection{Principles of Teaching Design for Elementary School Sci-} ence Curriculum Based on Immersive Virtual Reality Technology. Immersive virtual reality-based science classrooms facilitate effective learning to occur compared to traditional instructional classrooms. Immersive virtual reality classrooms are suitable for courses in which science content requires observational and inquiry-based experiments in which students need to observe and reflect on scientific phenomena to draw scientific conclusions. Dale's Tower of Experience theory suggests that the teaching and learning process should be filled with concrete experiences and gradually extend to abstract experiences. Learning activity theory suggests that real or simulated learning environments promote cognition and internalization by learners. Immersive virtual reality systems are based on a variety of perceptual technologies such as visual sensing, somatosensory and speech recognition, and haptic feedback to form an all-encompassing perceptual experience. It can render a realistic teaching environment through audio-visual elements such as light, pictures, sound effects, and colors, which is conducive to learners' active knowledge construction in an intuitive learning context and stimulates learners' keen interest in scientific research. Science classrooms are characterized by observation and experimentation to construct scientific concepts and form correct scientific values, so realistic natural scenes are crucial for knowledge understanding and memorization. Virtual reality technology as an emerging digital media technology needs to be analyzed more deeply, its characteristics to be characteristics, organize and summarize them, and study how they can be reasonably applied to the teaching field. In traditional classrooms, it is difficult for students to access real natural objects and landscapes, while virtual reality technology can use computers and other hardware devices to create highly simulated teaching situations, solving the problems of limited venue factors and high practical overhead in traditional classrooms. At the same time, students can be fully immersed in a teaching environment that is closely linked to the learning content, and they can perform repeated practice and gain experience to improve their scientific literacy.

To expand the computing function of the Internet, it is necessary to make full use of the "algorithm+" advantage brought by media fusion technology to master the characteristics of the Civic and Political Education object [22]. The development of media convergence has led the modern information society to gradually move toward the "era of mass media" and "era of intelligent media"; with the rise of various platforms driven by big data algorithms and virtual reality microcreations, several algorithms to capture, mine, and analyze data, combined with several personalized mobile platforms that combine data capture, mining, and analysis through algorithms, combined with visualization technology and user behavior such as reading, retweeting, and commenting data to accurately profile and push users have been developed and occupy a large amount of traffic. It shows that the computing function of the Internet is very effective in attracting users and increasing their stickiness. Therefore, college ideological and political educators should make full use of the algorithmic advantages of the fusion of big data and virtual reality, based on the intelligent recommendation engine of big data mining, to dig into the behavioral needs of educated people, master the development characteristics of the ideological and political education object, realize the personalized and accurate distribution of educational contents, and take the lead in content dissemination.

The virtual reality system mainly includes helmet display, headset, and data gloves and other related information input and output devices; virtual environment and software to complete the virtual space composition of the rule structure; computer processing system, image, and sound synthesis devices, etc. The main functional feature of virtual reality is its highly immersive experience. Students (people), virtual environments, virtual environment generators, role devices, and sensor devices are the main elements of the composition of common virtual reality systems; for example, Figure 1 shows the composition of virtual reality systems.

People use visual and auditory contained image information and sound information to create an interactive immersive experience, and the format has a unique cognitive model to immerse students from multiple senses. Human visual perceptual information sensing is the primary information reception channel in the multisensory and visual perception that has a more detailed information processing mode and interaction design by using the human visual information channel to input environmental and operational information and then immerse people in it. In modern product design, a large number of designers advocate adding an immersive experience mode, in which students can absorb information about product use through different senses and increase the pleasure in the process of using the product. For example, in puzzle games such as tower defense, the designer constrains the player's behavior by regulating the graphics, and the information is fed back to the brain to influence the student's behavior. Because visual perception has prophetic and timely transmission in human senses, its principles are applied in many fields, such as graphic art design, spatial art design, and software design, combined with the product design mode of immersive interactive experience. The application of visual perception principles in the design of immersive interactive experience can be 


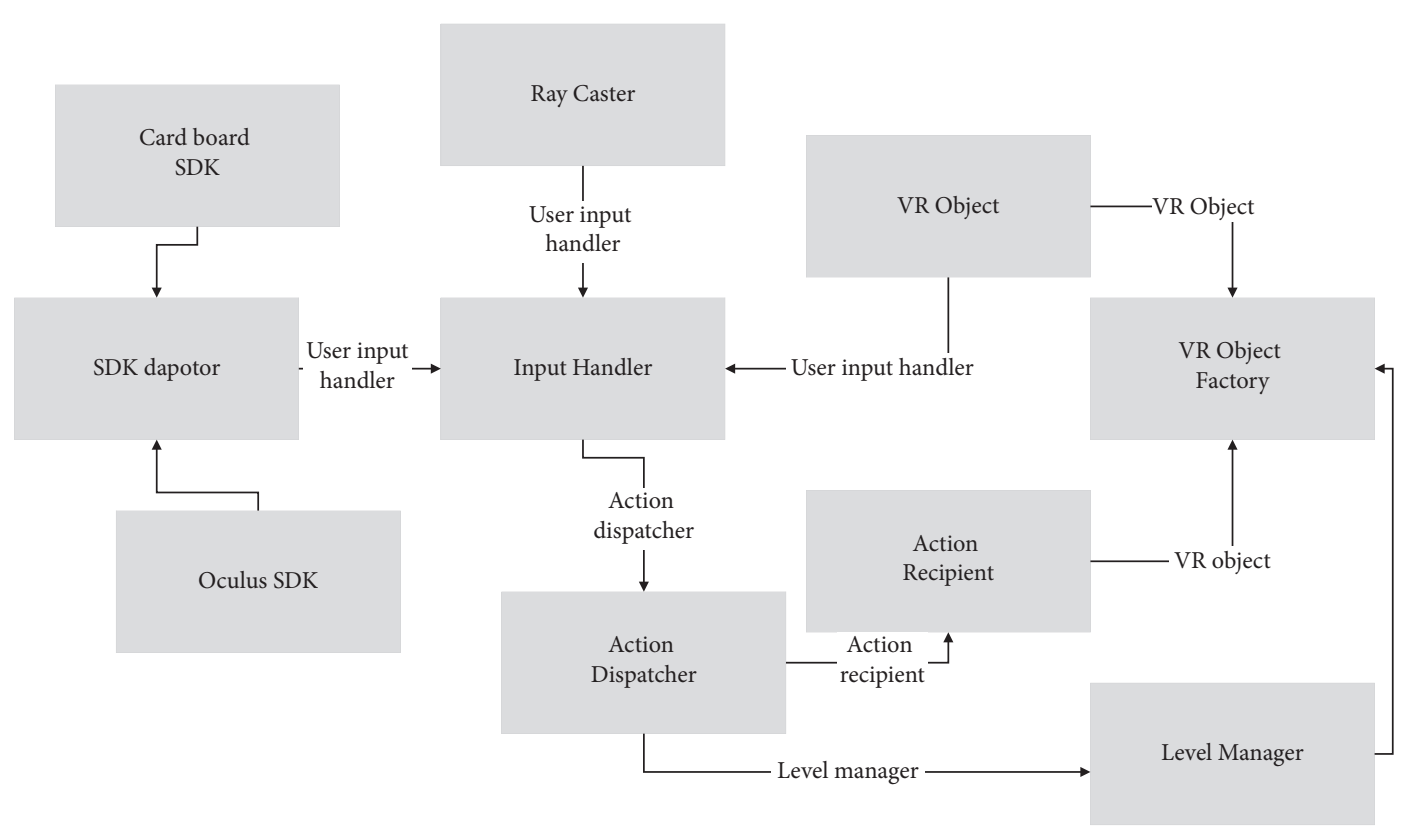

Figure 1: Composition of virtual reality system.

distinguished based on two major parts: visual reception and visual cognition and, in actual application, some product designs In practical application, some product designs only stay at the level of visual acceptance and visual stimulation, which is called "superficial application"; some product designs not only give students visual impact but also let them conduct cognitive analysis, which is called "deep application" here.

The five elements of the student experience are very similar to our daily product design process, which is conceptual design $>>$ functional design $>>$ information architecture and interaction design $>>$ interface design $>>$ visual design, and this process is also considered a standard process for product design and development [23]. Incorporating elements of student experience into this process can give the product a head start by providing basic student acceptance even before it goes live. A detailed analysis of the five student experience elements of the product is a process from the bottom to the top, from the conceptual framework to the interface visuals, gradually facing the student interface, and gradually reaching the experience of students, and its structural form is shown in Figure 2.

Students are curious about the world around them, and the virtual reality-based science classroom responds to this feature. Its graphic and realistic teaching environment creates a sense of auditory surroundings and immersion, which can quickly enhance students' interest and motivation for learning and help learners develop a relaxed and positive emotion and thus improve the learning effect [24-26]. The most critical technical point in the process of using virtual reality technology is to combine virtual environment, virtual sound effects, and visual information of space to synthesize a multidimensional digital information scenario. Situated cognition theory attempts to correct the failures of the symbolic-operational approach to cognition, especially cognition that relies exclusively on rules and information descriptions and cognition that focuses solely on conscious reasoning and thinking, ignoring the cultural and physical context. The science classroom based on immersive virtual reality technology can easily achieve human-computer interaction and interaction for all, making up for the interaction limitations of traditional multimedia teaching courseware. The immersive virtual reality-based science classroom has gamification features, which implement the educational concept of "teaching for fun" and enable students to feel the reality of scientific phenomena in lifelike dynamic scenes so that they can take the initiative to discover and ask questions. In addition, the communication and interaction between teachers and students help to strengthen the emotional perception and make the teaching atmosphere more relaxed and interesting, thus forming a good attitude toward science.

\subsection{Feedback Model of Virtual Reality Interaction in Teaching.} Emotional feedback is the expression of human attitudes toward external things or events. Emotional feedback governs the daily work of human beings, and emotional feedback usually has some correlation with personality, and in the process of virtual reality interactive education and teaching, emotional feedback can also be used to judge the experience of students in virtual reality. Emotional feedback is a subjective feeling, and it is usually necessary to establish an emotional feedback model to objectively define emotional feedback. Emotional feedback models can be divided into two models according to the continuous state: the first model is a continuous emotional feedback model, including the two-bit model of emotional feedback and the three-dimensional model of emotional feedback, as they express the intensity value of human emotional feedback in each 


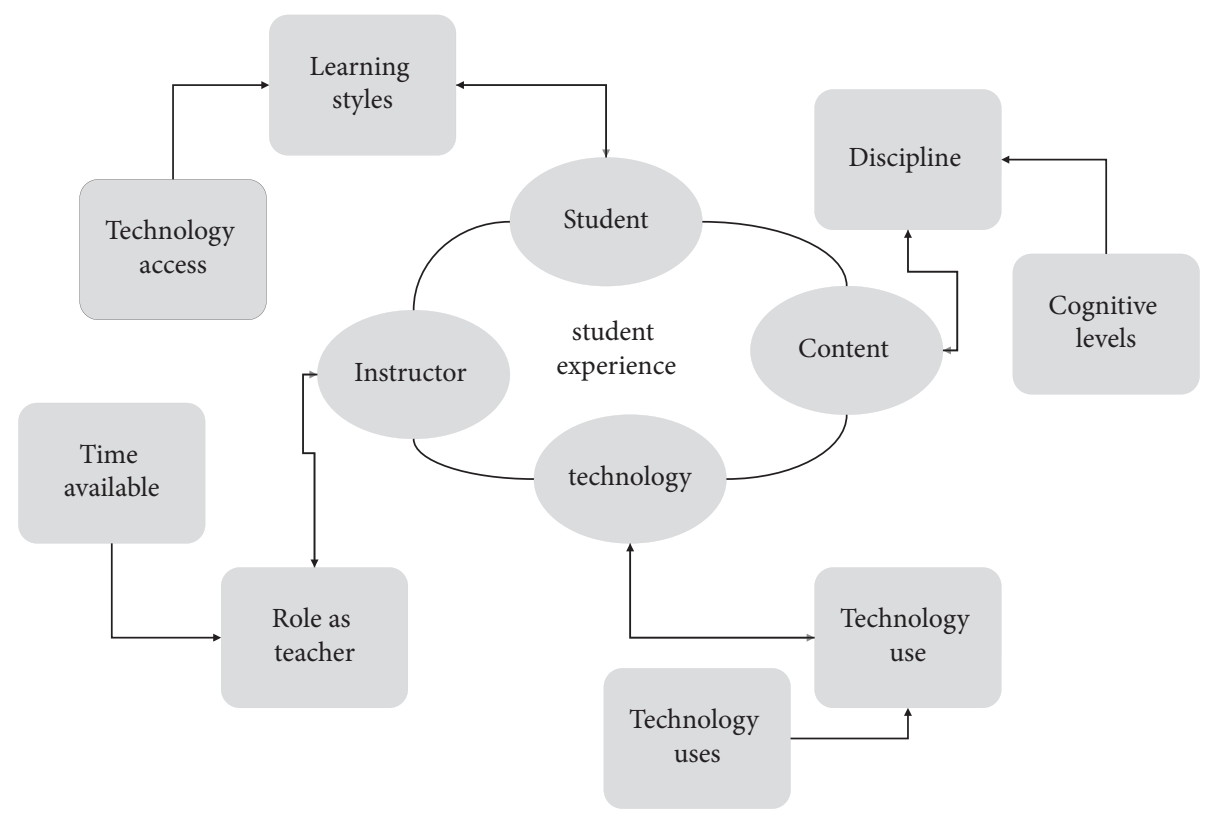

Figure 2: Aspects of the elements of the student experience.

dimension through different dimensions; the second model is a discrete model of emotional feedback; discrete models more specifically describe the type and state of emotional feedback. The discrete model includes happy, disappointed, and sad. By combining different dimensions and intensities of the continuous model, a discrete model of emotional feedback can be obtained. Emotional feedback plays an important role in human life, which can influence human thinking activities, decision-making ability, and interpersonal skills. Nonphysiological changes are usually reflected in facial changes and voice intonation changes; physiological changes are usually reflected in EEG signals and heart rate changes. Therefore, emotional feedback can be analyzed by capturing the subtle changes of humans. Emotional feedback can be represented in two or three dimensions, and research has shown that changes in emotional feedback are usually superimposed by changes in multiple dimensions of state. The two dimensions of emotional feedback are "pleasantness" and "arousal". "Pleasantness" represents the intensity of unpleasantness-pleasantness in response to something or an event; "arousal" represents the change of calm-excited state in response to something or an event. Both "pleasantness" and "arousal" are continuous values that can be represented by a continuous axis. Based on the two-dimensional model of emotional feedback, a three-dimensional model of emotional feedback emerges, as shown in Figure 3. The three-dimensional model of emotional feedback adds "dominance" to the two-dimensional model, and "dominance" expresses the degree of dominance of the human being over something or an event that produces a change in the state of emotional feedback, which is a change from tension to relaxation. See Figure 3.

Granger causality can analyze whether there is a causal relationship between two sets of data, so Granger causality is widely used in major research fields. Meteorologists use Granger causality to predict the weather, and

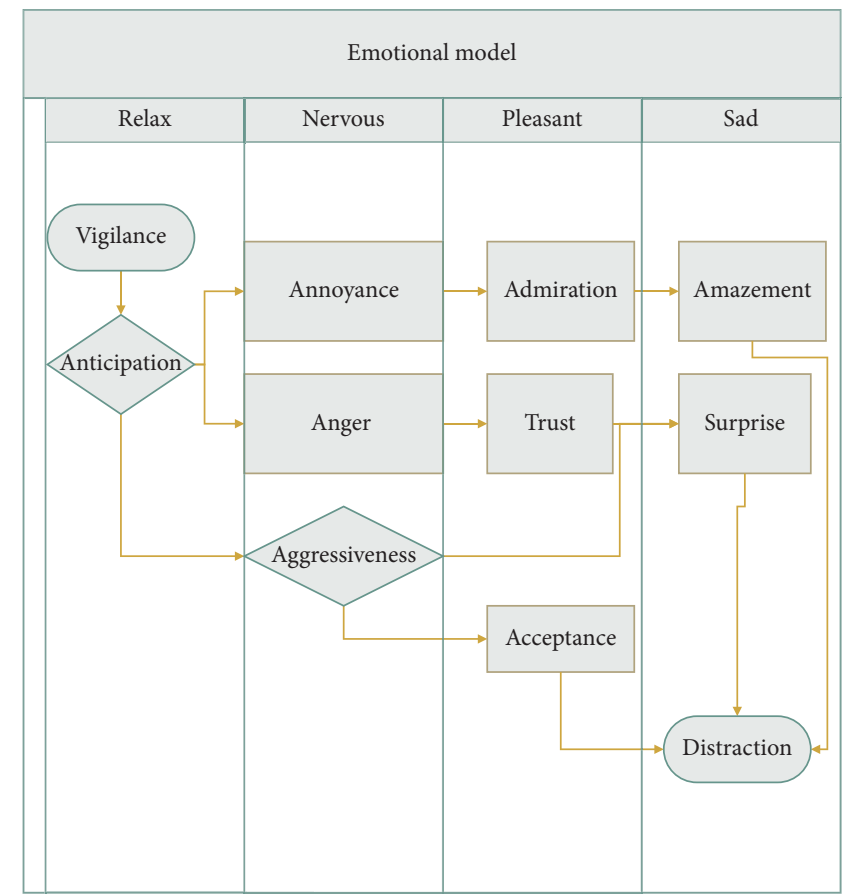

Figure 3: Emotional model.

neuroscientists use Granger causality to determine the causal relationship between physiological signals in different brain regions. Through the computer language to manipulate the user behavior of people in the process of using the product, the computer issued "The information command contains the listing and display of multidimensional information technology." Although, in the early stage, Granger himself opposed the application of Granger causality to other research fields, thinking that it was a "misuse" of Granger causality, after years of research and 
exploration, neuroscientists have found enough theoretical support to prove that Granger causality applies to the physiological electrical signals generated by neurons. It is due to the continuous attempts and innovations of the previous people that Granger causality can be "reasonably" applied to the field of brain science. Figure 4 shows the principle diagram of Granger causality.

The $X$ and $Y$ variables change over time. The purpose of Granger causality is to determine whether there is some correlation between $X$ and $Y$, i.e., whether the value of the $X$ variable affects the value of the $Y$ variable. This causal relationship is often used to predict the future values of variables; for example, when we predict the value of $Y$ variable, if $X$ variable has some relationship on the generation of $Y$ variable, then the present value of $X$ variable will be used as a predictor variable for the future value of $Y$. Clearly, there is bound to be some connection between anything. If the value of $X$ variable does not predict the future value of $Y$ variable as well as the past value of $Y$ variable alone, then it can be considered that the value of $X$ variable has no effect on the prediction of $Y$ variable and there is no Granger causality between $X$ variable and $Y$ variable. To perform Granger causality analysis of $X$ and $Y$ variables, we first need to establish their autoregressive models for $X$ and $Y$ variables, and the autoregressive model establishment formula is as follows:

$$
\begin{gathered}
X_{k}=\sum_{i=1}^{\kappa} \varepsilon_{i} X_{k-i}+C \gamma^{2}+D \eta^{1 / 2}, \\
Y_{m}=\sum_{i=1}^{\kappa} \varepsilon_{i}^{\prime} Y_{k-i}+C^{\prime} \gamma_{y}^{2}+D^{\prime} \eta_{y}^{1 / 2} .
\end{gathered}
$$

$p$ is the regression order of the autoregressive model, $A$ in equation (1) and $B$ in equation (2) are the autoregressive coefficients of variables $X$ and $Y, Z$ is the exogenous variable, and $\varepsilon$ and $\varepsilon^{\prime}$ are the residuals. From equation (1), it can be seen that the value of $X$ variable at moment $t$ can be calculated by the accumulation of the product of $\varepsilon$ moment values and autoregressive coefficients $A$ products before moment $t$ of $X$ variable, and the same is true for $Y$ series. When there is a causal relationship between the $X$ and $Y$ series, a joint regression model of $X$ and $Y$ can be developed as follows:

$$
\begin{aligned}
& X_{\kappa}=\int \frac{A x^{\gamma}}{B x^{1 / 2 \alpha}+C y} \mathrm{~d} x+D \eta+C, \\
& Y_{\kappa}=\int \frac{A^{\prime} y^{\gamma}}{B^{\prime} y^{1 / 2 \alpha}+C^{\prime} y^{3}} \mathrm{~d} y+D^{\prime} \eta_{y} .
\end{aligned}
$$

There are two approaches to determine whether there is Granger causality between the two series; the first approach is as described in the previous section: when either of $B$ in equation (3) or $A$ in equation (4) is not zero, then Granger causality is proved to exist between series $X$ and series $Y$. The second approach is to analyze the residuals from the autoregressive model and the joint model by using the following equation.

$$
\begin{aligned}
& F_{Y \rightarrow X}=\lg \left(\frac{X_{\kappa}}{Y_{\kappa}}+\alpha X_{k}-\beta Y_{m}\right)+\frac{C}{C^{\prime}}, \\
& F_{X \longrightarrow Y}=\lg \left(\frac{Y_{\kappa}}{X_{\kappa}}+\varepsilon^{\prime} Y_{m}-\varepsilon X_{k}\right)+\frac{C^{\prime}}{C} .
\end{aligned}
$$

The brain is composed of hundreds of billions of neuronal cells, and neurons in different areas coordinate, interact, and transmit information to each other. When a task needs to be accomplished, information is processed, exchanged, and transmitted between neurons in each neuron's brain region and neurons in other functional regions, which together control human behavior such as feelings, thinking, speech, and action. Therefore, studying the information transfer relationship between the subject's brains can tell the students' emotional feedback about teaching in different virtual reality scenarios and thus the role that virtual reality interactive teaching produces in education.

\section{Experimental Design and Validation}

The immersive virtual reality classroom was built mainly using 3 ds Max software for modeling the basic model and giving the materials, and Pano2VR was used to convert the virtual classroom into panoramic mode. The virtual reality classroom built this time did not achieve real interaction; after all, a completely immersive virtual reality environment requires a technically mature $R \& D$ team. Before building a virtual reality classroom, it is important to be clear about what objects are in the classroom, how they are placed, what the size of the specifications is, and what the specific values of the length, width, and height of the classroom are, which is the prep work for conducting a basic scene modeling. By modeling the virtual classroom concerning a real classroom, students can be less distracted and more attentive to their learning when they are in a real teaching situation. Virtual reality classrooms can increase student motivation and promote the development of scientific thinking. The powerful interactive capabilities of virtual reality technology deliver operations to the virtual teaching environment in a natural way and in real-time, enabling immersive learning activities. The process of learning through the interaction of different perceptual systems in the student's psyche is to establish learning objectives to give people a different sensory experience and cognitive experience to attract users which is a way of display design. Learners use input devices such as operating handles or data gloves to form a time series of learning behaviors and then use the human-computer interaction interface to transfer the data to the computer, enabling analysis of student learning behaviors and giving operational scores to form data feedback. Students can consolidate their knowledge by checking for gaps based on detailed scores. Teachers can also log into the learning management system to inquire about students' learning and analyze the important and difficult points in teaching through visual data, to improve the teaching 


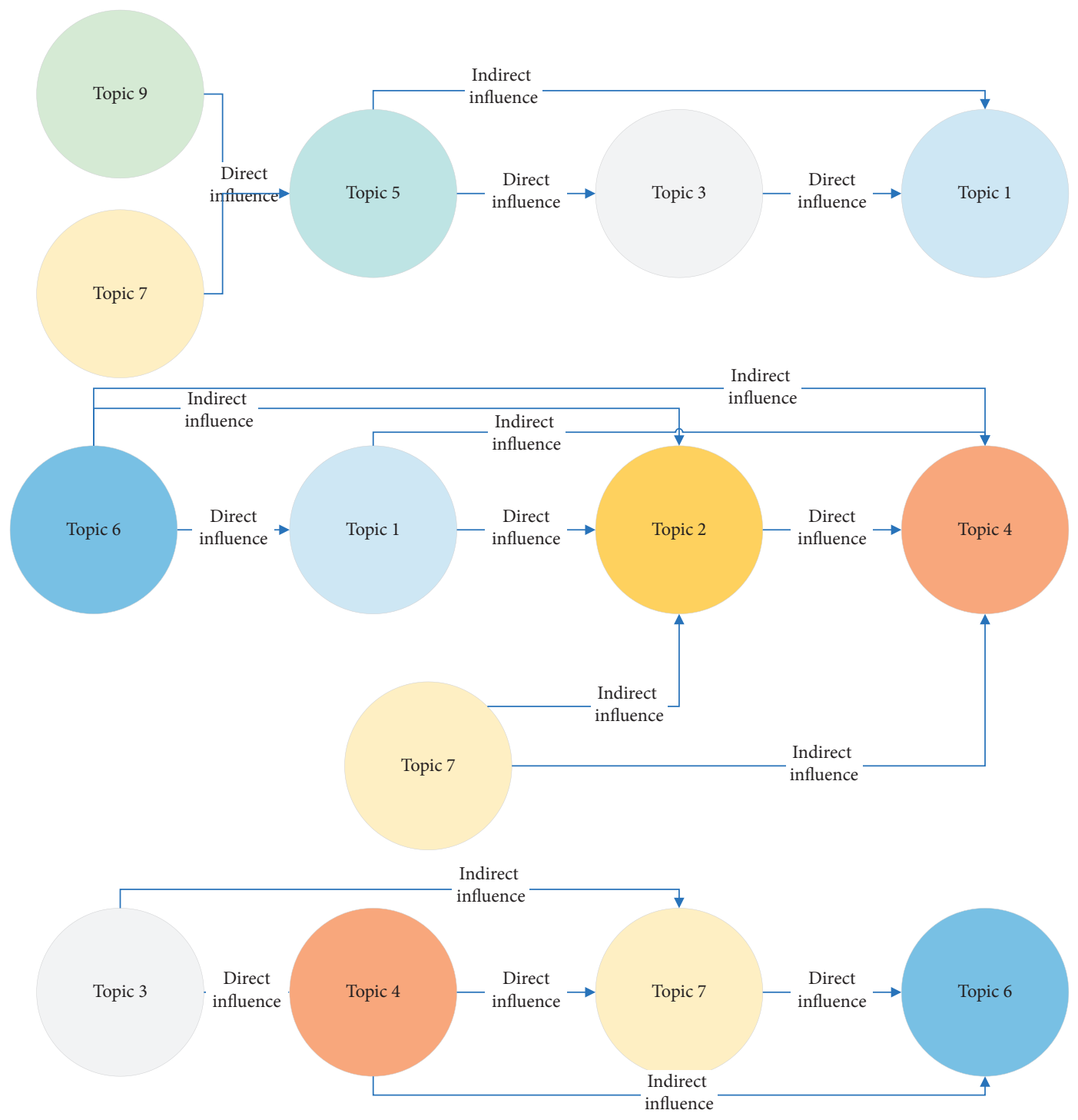

Figure 4: Granger causality diagram.

process as well as teaching methods in a targeted manner and enhance the quality of teaching.

Students were first asked to view the generated dynamic VR panoramic virtual classroom map through the web version and choose their observation angle in full-screen mode by going up, down, left, right, zoom in, zoom out, and autorotate to roam around the virtual reality classroom to personally experience and feel the visual impact of the immersive virtual reality classroom. After experiencing the immersive virtual reality classroom, we get the students' emotional feedback by comparing it with the real physical classroom. We conduct teaching and learning activities with virtual reality technology and perform teaching effectiveness analysis, including classroom interaction analysis and learning tests.

In an immersive virtual reality science classroom, teachers design the flow of instruction based on learning activities. The learning time of the virtual reality courseware should not be too long; otherwise, the learning subjects are prone to vertigo and reduce the learning efficiency. In this paper, the EEG data of 20 subjects were taken, each subject watched $10 \mathrm{VR}$ scenes, and a total of 200 sample data were collected. Based on each subject's emotional 3D scale data, samples with arousal greater than 5 were designated as positive samples, samples with arousal less than 5 were designated as negative samples, and the ratio of positive to negative samples was $96: 104$. Due to the small sample size, to get more sample data, this paper divided a single sample into 10 small samples, the expanded sample size was 2000, and many studies in the past have also applied this. This method has been used in many past studies to expand the sample size. Since the state of the brain affected by the scene is a sustainable process under a single scene, the labels of the expanded sample are kept consistent with the original sample. The above features are identified by extracting four time domain features of EEG data: energy value, standard 
deviation, first-order difference, and differential entropy, respectively. 10-fold cross-validation was used for the identification of each feature, and the average results of 10 cross-validations were obtained. As shown in Figure 5, the recognition rates of the features are all kept around $80 \%$, among which the differential entropy feature has the highest recognition rate of $80.18 \%$, but the recognition rate of firstorder difference as a feature for classification is not high, only $63.15 \%$. The reason for the poor effect of first-order difference may be that the first-order difference is the incremental size between two data points before and after, and the difference between the values of two adjacent data points may not be very large, so the first-order difference only reflects the local characteristics of EEG data. In the interaction design process, we should try to reduce students' cognitive load and reduce the cost and time for students to think during operation, i.e., let students choose the next operation task. Differential entropy and other features reflect the overall characteristics of EEG data, and the gap between sample features is relatively large. Figure 5 shows the accuracy histogram drawn from the results of 10 times crossvalidation. The height of the bar chart is equal to the average of the 10 cross-validations, and the horizontal line above the bar chart represents the variance value. It can be seen that the accuracy variance of differential entropy is smaller than the accuracy variance of energy value, and the 10-time recognition accuracy of differential entropy is more stable and does not change too much due to the change of training samples.

Overly simple operations tend to cause boredom and boredom in the learning subject, while overly difficult operations cause worry and anxiety in the learning subject, leading to a decrease in learning efficiency. In addition, students can get feedback and suggestions from the interaction sessions and adjust their learning plans in time. In the virtual reality environment, there are various forms of interaction, including question-and-answer interaction, which allows students to review and consolidate existing knowledge and promote reflection activities; tactile manipulation, which allows students to immerse themselves in the world of science and enhance the sense of reality and immersion; and experimental investigation, which allows students to gain a deeper and more comprehensive understanding of scientific phenomena and processes and improve their hands-on skills. To study the differences in recognition rates of each frequency band, the data are decomposed into four frequency bands, and PSD features are extracted for the full-frequency signal and each sub-band signal, respectively. The recognition rate of the Gamma-band is the highest among all subbands, which shows that the relevance of high-frequency signals for arousal is higher than that of low-frequency signals. The recognition rate of the full-frequency signal is $80.06 \%$. The correlation of the full-frequency signal for wakefulness is closer than that of the sub-bands, which may be related to the fact that the full-frequency signal contains the information of the frequency bands with each other. From Figure 6, we can see that the variance of the full-frequency feature accuracy is smaller, and the

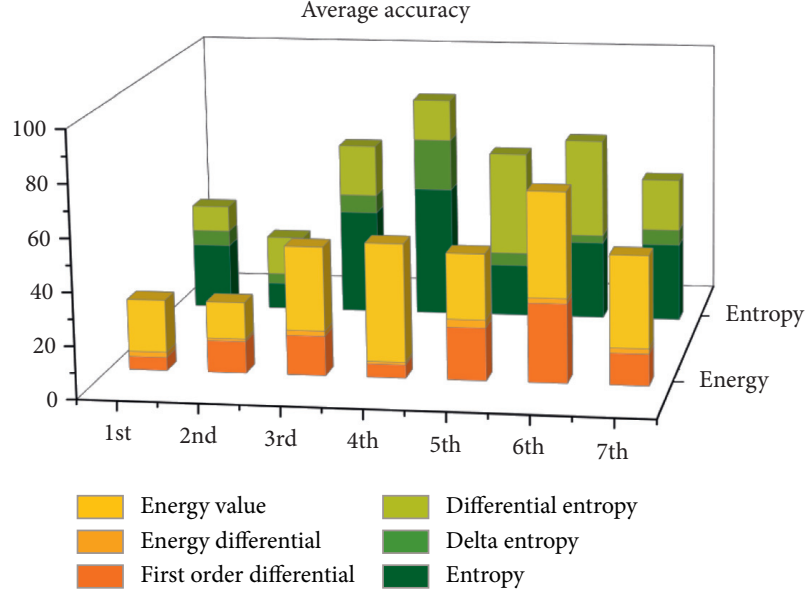

Figure 5: Time domain feature classification results.

recognition results of 10 times cross-validation are more stable and do not change too much due to the change of training samples.

Effective assessment promotes learning activities and detects whether learning subjects have completed their learning tasks and achieved their learning goals. Learning evaluation has a diagnostic function, which can accurately reflect the learning status of the learning subject and understand the degree of accomplishment of learning objectives; learning evaluation has a regulatory function, which can make the learning subject recognize the shortcomings in learning and thus promote the occurrence of efficient learning; learning evaluation has a motivational function, which has a supervisory and reinforcing effect on teachers and learning subjects. The time domain features and frequency domain features are fused, and it is analyzed whether the recognition accuracy is improved after feature splicing and fusion. The application in immersive interaction design for virtual reality is as follows. The visual perception of information can maximize the effect of deep graphical information knowledge learning for students. The features extracted from the four subfrequency bands were combined two by two and classified using the method described above, with the kernel function being RBF and all parameters tuned to optimality. The classification accuracies of the frequency group associated with theta band were all relatively high, reflecting the high correlation between theta wave and arousal degree, and theta wave indicates that the brain is in the thinking state. It can be seen from the comparison that the classification recognition rate of theta wave alone does not differ much from the resolution in the band group, and it can be presumed that theta wave dominates in the band group, and the recognition effect of theta band features is more stable; the classification recognition rate of gamma-band decreases significantly, and it can also be presumed that the dominance of gamma-band features in the full-frequency signal is small compared with other frequency bands. The recognition effect of gamma-band in the band group is "diluted" by other bands in the group. 


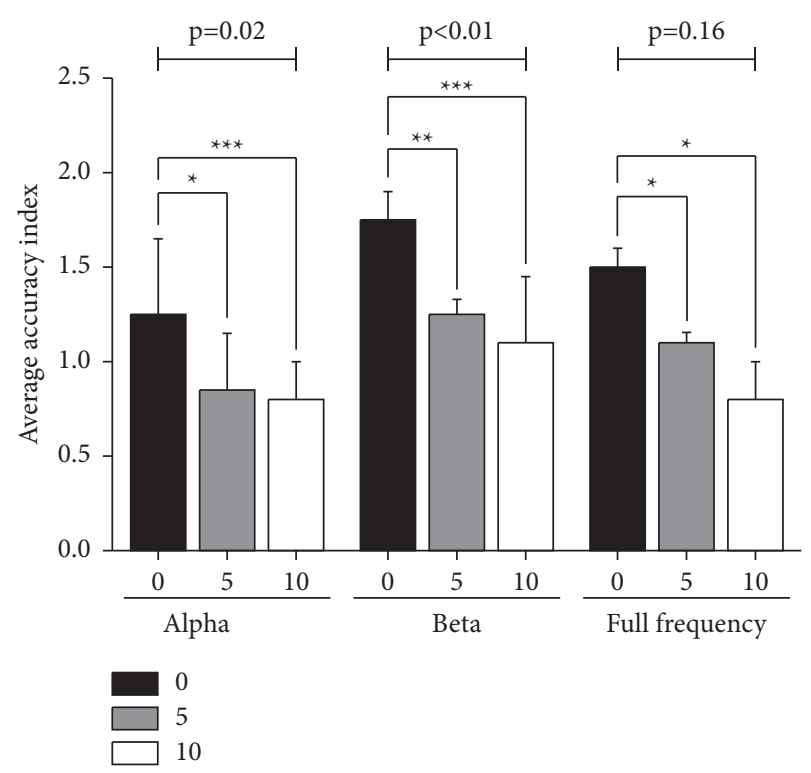

FIGURE 6: Classification results of spectral features.

The features of each subfrequency domain are combined and analyzed for recognition. In the following, the time domain and frequency domain features are combined, and the samples obtained after the combination of time domain and frequency domain are classified and recognized. In the classification recognition results, the classification recognition rate in three cases of time domain, frequency domain, and time-frequency all reach more than $80 \%$, among which the arousal degree classification recognition rate of time-frequency features is $82.33 \%$, and the recognition rate of time-frequency features has a great improvement compared with the classification recognition rate of time domain and frequency domain alone. According to the above results, this paper uses the PCA algorithm to fuse the combined time-frequency features and project them to the low-dimensional space and recognize them separately. As in Figure 7, the horizontal coordinate is the dimension of the feature, and the vertical coordinate is the classification recognition rate of the feature in that dimension.

In this learning process, students not only master the theoretical knowledge but also can have a series of interactions with the virtual environment and practical experience of what the teacher has taught them in the interactive process, which will also make that part of knowledge forever remembered in the students' minds. Not only that, it is impossible for human creativity to appear by imagination alone, but it must be personally practiced in life and inspired by practice, thus leading students to design innovation. In another way, the practice within the virtual reality environment is situational in its activity. In addition to acquiring specific information, students gain silent knowledge that is not available in traditional learning models through a number of their activities (body movements, brain musings, etc.). The characteristic points exhibited by virtual reality can give practical meaning to the

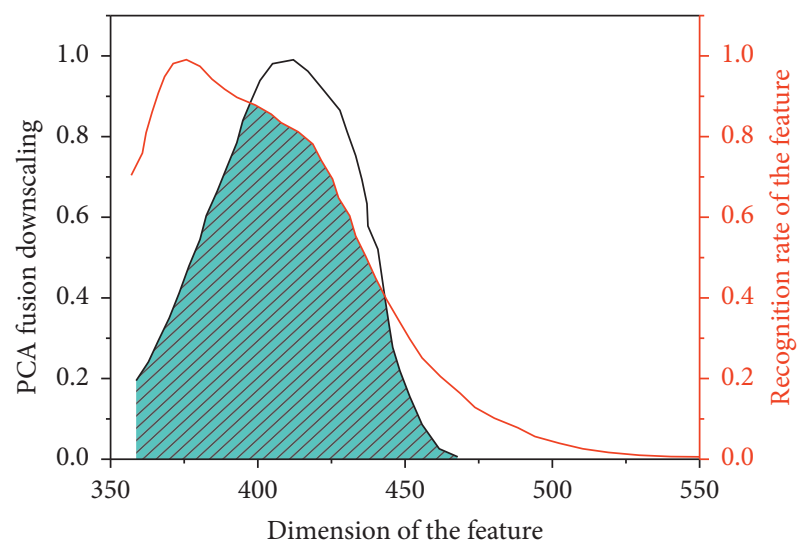

FIgURe 7: PCA fusion downscaling.

student's learning process, addressing many of the limitations of practicing in prototypical conditions through virtual reality technology. Although virtual exercises are still based on objective reality, once students enter the virtual interior space, they are no longer limited by operations that are difficult to practice in a realistic environment. Instead, they can integrate the theoretical knowledge previously taught by the teacher into "predefined" scenarios and visions and analyze and validate a large amount of realistic material through virtual technology to find and discover the possibilities of new situations in interior design. In this way, the virtual and real poles are organically united in the new technological practice. By studying Civics in virtual space, students will learn a complete, situational, and full knowledge including silent knowledge, not just skeletal abstract explicit knowledge.

The display medium in the virtual world will no longer be a single flat surface, and the display range will not be limited. The design freedom at this point is extremely high, and the three-dimensional icons are more recognizable in the virtual environment but also combined with a threedimensional environment that is more realistic for the user to experience when touching or clicking. A comparison of the behavioral ratios of the four major components of the VR classroom and the traditional classroom is shown in Figure 8.

Based on the analysis, it can be concluded that before the teaching experiment, the students' motivation level was average and did not show significant passion. After one semester of the teaching experiment, students' overall motivation to learn produced a significant increase, indicating that the immersive virtual reality-based classroom was able to stimulate students' interest in learning. There was some improvement in student attention dimensions compared to the traditional teaching approach, but the variability change in the pre-post test comparison was not as large as the pre-post change in the experimental group. In summary, it shows that the virtual reality-based classroom is more capable of increasing students' interest in learning than the traditional teaching classroom. 


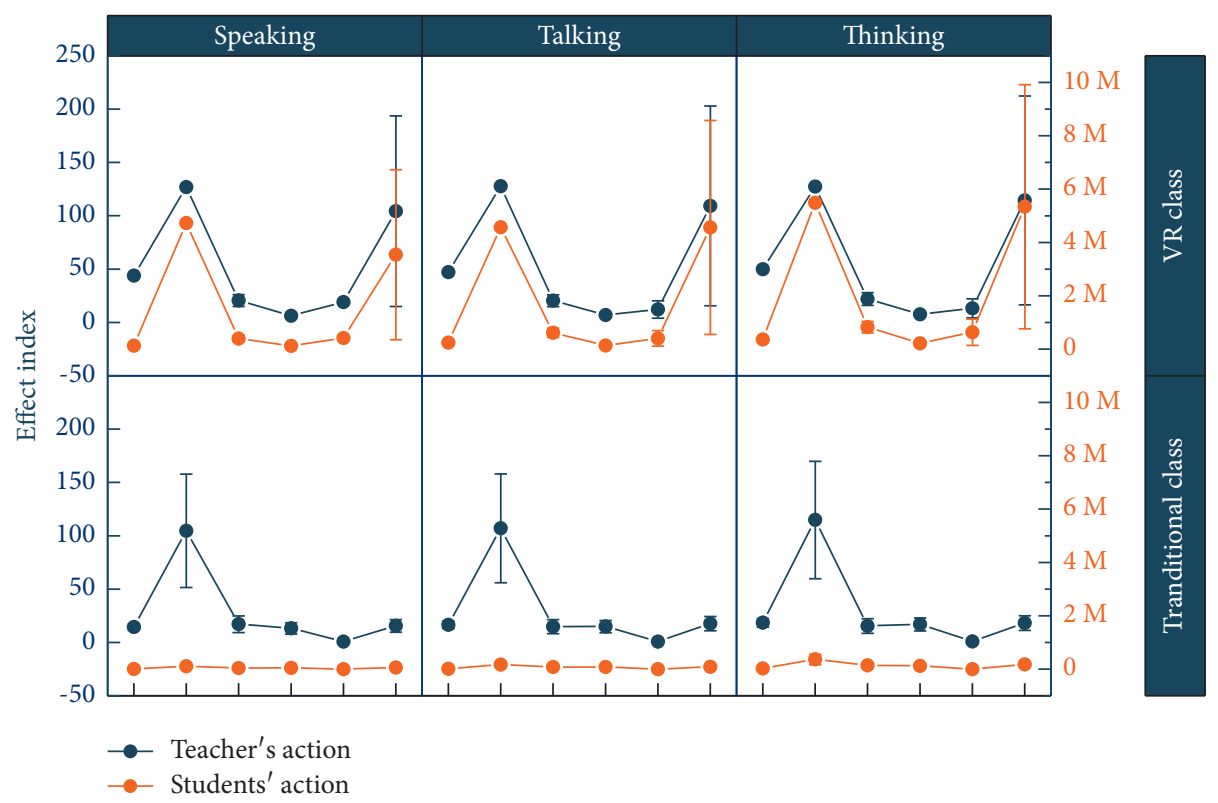

FIGURE 8: Discounted VR class vs. traditional class interaction behavior.

\section{Conclusion}

This paper presents a virtual reality-based interactive learning system for secondary school students based on the principles of virtual reality technology and combines various design theories to analyze and organize the virtual reality interaction in the Civics course in the big data environment, find teaching problems, and then transform the problems into design ideas. Through the application development and design of the biology learning module for secondary school students as an example and usability evaluation and testing to verify the usability and applicability of the system in secondary school students learning, virtual reality technology has a variety of innovative forms of interaction, most of which have serious technical barriers, and virtual reality where this technology has not yet been perfected also has greater development space, for the development of interactive experience form has also a significant role; perhaps in the future, these unachievable forms of interaction can become a reality. Based on a variety of design theories and technology exploration, the system's design methods and design principles are proposed based on theories of ergonomics, human-computer interaction, user experience, and immersive learning by analyzing and organizing the needs in the Civics course learning in-depth; the interactive learning system for students in the virtual reality context in this topic integrates sound, images, animation, audio, and rich $3 \mathrm{D}$ models and other multimedia learning resources and presents these learning resources in the form of virtual reality in front of students. The high degree of immersion and rich interactive forms provide students with a high-tech teaching mode, fully demonstrating the advantages of virtual reality in future education.

\section{Data Availability}

The data used to support the findings of this study are included within the article.

\section{Conflicts of Interest}

The authors declare that they have no conflicts of interest.

\section{References}

[1] Y. Falaq, "Education of citizenship in higher education as A fortress of nation characters in facing era society 5.0," Journal of Educational Sciences, vol. 4, no. 4, pp. 802-812, 2020.

[2] N. Dvir and O. Schatz-Oppenheimer, "Novice teachers in a changing reality," European Journal of Teacher Education, vol. 43, no. 4, pp. 639-656, 2020.

[3] R. Sivaraj, J. A. Ellis, J. R. Wieselmann, and G. H. Roehrig, "Computational participation and the learner-technology pairing in K-12 STEM education," Human Behavior and Emerging Technologies, vol. 2, no. 4, pp. 387-400, 2020.

[4] E. G. Rincón-Flores, J. Mena, and M. M. S. R. Gamification, "A new key for enhancing engagement in MOOCs on energy?" International Journal on Interactive Design and Manufacturing, vol. 14, no. 4, pp. 1379-1393, 2020.

[5] G. Dishon, "The new natural? Authenticity and the naturalization of educational technologies," Learning, Media and Technology, vol. 46, no. 2, pp. 156-173, 2021.

[6] S. Schroeder, R. Curcio, and L. Lundgren, "Expanding the learning network: how teachers use Pinterest," Journal of Research on Technology in Education, vol. 51, no. 2, pp. 166-186, 2019.

[7] N. F. Handayani and A. Gafur, "Professionalism of civics teachers facing educational challenges in the era of the covid19 pandemic," International Journal of Multicultural and Multireligious Understanding, vol. 7, no. 10, pp. 527-534, 2020.

[8] M. Ghosn-Chelala, "Exploring sustainable learning and practice of digital citizenship: education and place-based challenges," Education, Citizenship and Social Justice, vol. 14, no. 1, pp. 40-56, 2019.

[9] M. Zid, S. N. Hijrawadi, F. Ramadhoan, and A. R. Casmana, "The preparation of the social science teacher in jakarta senior 
high school in facing the industrial revolution 4.0," Education, vol. 29, no. 4, pp. 4572-4589, 2020.

[10] L. W. Peng and C. Y. Lee, "Virtualizing science to maximize self-efficacy, value, and motivation for tomorrow's science workforce," Excellence in Education Journal, vol. 9, no. 3, pp. 49-81, 2020.

[11] R. Reynolds, S. Macqueen, and K. Ferguson-Patrick, "Active citizenship in a global world: opportunities in the Australian Curriculum," Curriculum Perspectives, vol. 40, no. 1, pp. 63-73, 2020.

[12] E. G. R. Flores, J. Mena, M. S. R. Montoya, and R. R. Velarde, "The use of gamification in xMOOCs about energy: effects and predictive models for participants' learning," Australasian Journal of Educational Technology, vol. 36, no. 2, pp. 43-59, 2020.

[13] M. Yunus, P. Setyosari, S. Utaya, and D. Kuswandi, "The influence of online project collaborative learning and achievement motivation on problem-solving ability," European Journal of Educational Research, vol. 10, no. 2, pp. 813-823, 2021.

[14] G. J. Hwang, K. C. Li, and C. L. Lai, "Trends and strategies for conducting effective STEM research and applications: a mobile and ubiquitous learning perspective," International Journal of Mobile Learning and Organisation, vol. 14, no. 2, pp. 161-183, 2020.

[15] E. O. Crawford, H. J. Higgins, and J. Hilburn, "Using a global competence model in an instructional design course before social studies methods: a developmental approach to global teacher education," Journal of Social Studies Research, vol. 44, no. 4, pp. 367-381, 2020.

[16] J. A. Chen and J. D. Stoddard, "A virtual internship to prepare high school students for civic and political action," Educational Technology Research \& Development, vol. 68, no. 6, pp. 3449-3470, 2020.

[17] J. B. Kwon, K. DeBruler, and K. Kennedy, "A snapshot of successful K-12 online learning: focused on the 2015-16 academic year in Michigan," Journal of Online Learning Research, vol. 5, no. 2, pp. 199-225, 2019.

[18] D. Rosengrant, P. Money, T. Beyer, and B. Alexander, "Video game vignettes and more in the classroom," The Physics Teacher, vol. 57, no. 9, pp. 604-607, 2019.

[19] A. Garcia and R. S. De Roock, "Civic dimensions of critical digital literacies: towards an abolitionist lens," Pedagogies: International Journal, vol. 16, no. 2, pp. 187-201, 2021.

[20] A. Marin, K. H. Taylor, B. R. Shapiro, and R. Hall, "Why learning on the move: intersecting research pathways for mobility, learning and teaching," Cognition and Instruction, vol. 38, no. 3, pp. 265-280, 2020.

[21] J. L. Kenna and M. A. Hensley, "Utilizing social media to promote civic engagement in the social studies classroom," The Social Studies, vol. 110, no. 2, pp. 86-94, 2019.

[22] S. J. Kaka, J. Littenberg-Tobias, T. Kessner et al., "Digital simulations as approximations of practice: preparing preservice teachers to facilitate whole-class discussions of controversial issues," Journal of Technology and Teacher Education, vol. 29, no. 1, pp. 67-90, 2021.

[23] S. Piasecki, "Gamification in educational contexts," International Journal of Advanced Pervasive and Ubiquitous Computing, vol. 11, no. 2, pp. 41-67, 2019.

[24] C.-C. Huang, "User's segmentation on continued knowledge management system use in the public sector," Journal of Organizational and End User Computing, vol. 32, no. 1, pp. 19-40, 2020.
[25] N. Ramu, V. Pandi, J. D. Lazarus, and S. Radhakrishnan, “A novel trust model for secure group communication in distributed computing," Journal of Organizational and End User Computing, vol. 32, no. 3, pp. 1-14, 2020.

[26] A. O. Aderonke, U. Musa, and S. Oni, "E-revenue adoption in state internal revenue service: interrogating the institutional factors," Journal of Organizational and End User Computing, vol. 32, no. 1, pp. 41-61, 2020. 